\title{
Novel frameshift mutations in DSPP cause dentin dysplasia type II
}

The current classification system of hereditary dentin defects was proposed in 1973 (Shields, Bixler, \& el-Kafrawy AM, 1973) based on clinical and radiographic information without knowledge of the underlying molecular pathophysiology: three types of dentinogenesis imperfecta (DGI-I, DGI-II, and DGI-III) and 2 types of dentin dysplasia (DD-I and DD-II).

Dentin sialophosphoprotein (DSPP) is the most abundant non-collagenous component in dentin and a member of the acidic secretory calcium-binding phosphoprotein (SCPP) gene family (Kawasaki \& Weiss, 2006). After DSPP is synthesized, it undergoes extensive post-translational modifications (Yamakoshi, Nagano, $\mathrm{Hu}$, Yamakoshi, \& Simmer, 2011) and is cleaved into two major functional units: an $\mathrm{N}$-terminal fragment called dentin sialoprotein (DSP) and a C-terminal fragment known as dentin phosphoprotein (DPP) (Yamakoshi \& Simmer, 2018). Mutations in DSPP have been identified to cause DGI-II, DGI-III, and DD-II (Kim \& Simmer, 2007), and there are some overlapping phenotypes in some cases (McKnight, Simmer, Hart, Hart, \& Fisher, 2008). Therefore, it has been suggested that these three diseases are not separate entities but a spectrum of the disease depending on the degree of severity (Beattie et al., 2006).

A genotype-phenotype correlation with regard to the frameshift mutations in the DPP region was suggested when $\mathrm{N}$-terminal frameshifts in the DPP region were observed in association with DD-II, and more C-terminal frameshift mutations were found to cause DGI-II (McKnight, Suzanne Hart, et al., 2008). DD-II like frameshift mutations would generate shorter negative charged repeats than DGI-II like mutations in the $\mathrm{N}$-terminus of the DPP. A reduction of the interaction of the mutant DSPP in the ER with the wild-type DSPP through the $\mathrm{Ca}^{2+}$ bridge in the DD-II like mutation, enabling the secretion of the wild-type DSPP into the dentin matrix, was suggested as a molecular basis of the genotype-phenotype correlation (von Marschall, Mok, Phillips, McKnight, \& Fisher, 2012).

In this study, we recruited four families with DD-II and performed a mutational analysis, including Sanger sequencing of exons and exon-intron boundaries of DSPP and cloning of DPP repetitive sequence. The novel mutation identified in families 1 and 2 (Turkish families) [c.2134delA, p.(Ser712Alafs*602)] confirms the previous genotype-phenotype correlation and extends the range about 70 bp down to the C-terminus (Figure 1). The previous exception to the correlation was a DD-II family caused by the c.3135delC [p.(Ser1045Argfs*269)] mutation (Yang et al., 2016), and there were some features of DGI-II such as slight discoloration, bulbous crowns, and obliterated pulp chambers in some teeth. Interestingly, family 3 (Korean family) has an overlapping phenotype with a slight discoloration in the lingual side of the anterior teeth in the proband on the DD-II phenotype, and the location of the mutation [c.3480_3481insCTGCT, p.(Asp1161Leufs*155)] is similar to a previous family. Family 4 (Korean family) has an extremely mild, characteristic DD-II phenotype, and the mutation [c.3179delG, p.(Ser1060Thrfs*254)] is also close to the above 2 families. This study confirms the previous genotype-phenotype correlation and extends the range of the DD-II-associated N-terminus of the DPP region; however, it also provides additional exceptions to the previously DGI-II-associated C-terminus of the DPP region. 
(a)
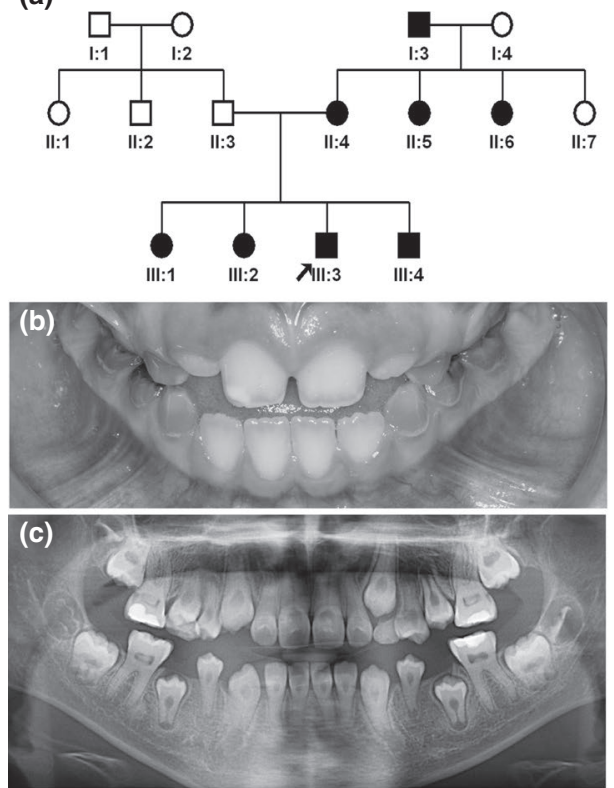

(d)

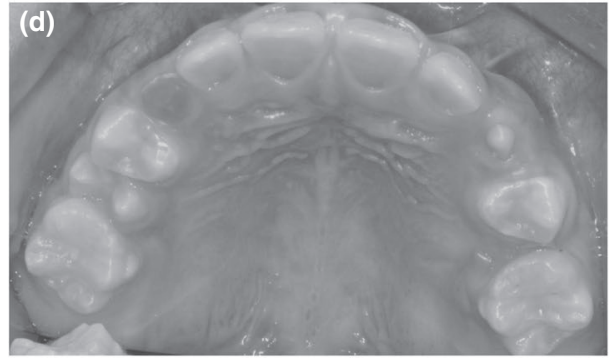

(e)

Family 1

GTGATAGTGACAGCAGTGATAGT GTGATAGTGACGCAGTGATAGTA

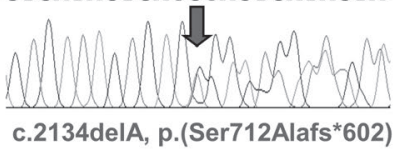

Family 2

GTGATAGTAGTGACAGCAGTGAT GTGATAGTGACGCAGTGATAGTA

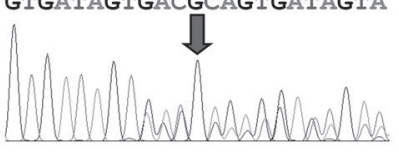

c.2134delA, p.(Ser712Alafs*602)

Family 3

AGCAGCGATAGCAGCGACAGCAG

TGACAGCAGCCTGCTGACAGCAG

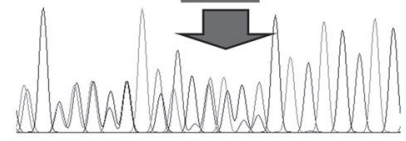

c.3480_3481insCTGCT

p.(Asp1161Leufs*155)

Family 4

AGCAGTGACAGCAGCGACAGCAG wt

AGCAGTGACACAGTGACAGCAGC mut

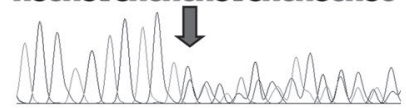

c.3179delG, p.(Ser1060Thrfs*254) wt

mut

wt

mut

wt

mut

(f)

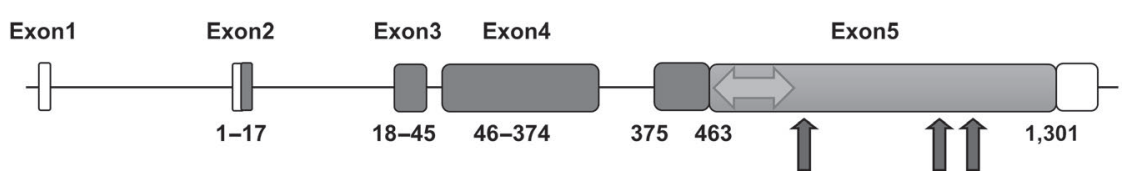

FIGURE 1 Pedigree, clinical photograph, panoramic radiograph, sequencing chromatograms of family members and gene diagram of DSPP. (a) Pedigree of family 1. (b) Clinical photograph of the proband (III:3) at the age of 8 years. Remaining deciduous teeth show an amberbrown discoloration and mild to moderate attrition, but erupting permanent teeth look normal without any discoloration. (c) Panoramic radiograph of the proband at the age of 10 years reveals the characteristic thistle tube-shaped pulp chambers with pulp stones. (d) Clinical photograph of the proband of family 3 at the age of 9 years 8 months. Remaining deciduous teeth exhibit a dark brown discoloration and severe attrition. Permanent dentition is normal in shape and color in most teeth, but the lingual surfaces of the maxillary anterior teeth show a mild brown hue at the cervical area. (e) Sanger sequencing chromatograms of the mutations identified. Wild-type (wt) and mutant (mut) nucleotide sequences are written on the above each chromatogram. The location of the mutations (deletion and insertion) was indicated with a red arrow in each chromatogram. Sequences based on the reference sequence for mRNA (NM_014208.3), where the A of the ATG translation initiation codon is nucleotide 1. (f) DSPP consists of 5 exons. Boxes indicate exons, and the amino acid numbers encoded by the exon are shown below each exon. The white area indicates the non-coding part, and the blue area indicates DSP region. Orange color indicates DPP region (463-1301 amino acids). The area associated with DD-II is shown as a green double arrow. The red arrows indicate the position of identified mutations in this study [Colour figure can be viewed at wileyonlinelibrary.com]

\section{ACKNOWLEDGEMENTS}

We thank the participants in this study for their cooperation. This work was supported by the National Research Foundation of Korea (NRF) grant funded by the Korean government (NRF-2018R1A5A2024418) and by NIDCR/NIH grant 1R01DE27675. The authors declare no potential conflicts of interest with respect to the authorship and/or publication of this article.

\section{CONFLICT OF INTEREST}

The authors declare no conflict of interests.

\section{AUTHOR CONTRIBUTIONS}

J.W. Lee and J. Hong contributed to analysis and interpretation, drafted the manuscript, and critically revised the manuscript; F. 
Seymen, M. Koruyucu, N. Tuloglu, S. Bayrak, and J.-C. Lee contributed to the conception, design, and data acquisition, and critically revised the manuscript; Y.J. Kim and J. Kang contributed to data analysis and interpretation, and critically revised the manuscript; J.-S. Song, T.J. Shin, H.-K. Hyun, Y.-J. Kim, and J.-C. Park contributed to the conception and critically revised the manuscript; and J. Hu, J. Simmer, and J.-W. Kim contributed to the conception, design, data acquisition, analysis, and interpretation, drafted the manuscript, and critically revised the manuscript. All authors gave final approval and agree to be accountable for all aspects of the work.

\section{Ji Won Lee ${ }^{1}$ Jiwon Hong ${ }^{1}$ Figen Seymen ${ }^{2}$ Youn Jung $\mathrm{Kim}^{3}$ Jenny Kang ${ }^{1}$ Mine Koruyucu ${ }^{2}$ Nuray Tuloglu ${ }^{4}$ Sule Bayrak ${ }^{4}$ Ji-Soo Song ${ }^{1}$ Teo Jeon Shin ${ }^{1}$ Hong-Keun Hyun ${ }^{1}$ Young-Jae Kim ${ }^{1}$ Jae-Cheoun Lee ${ }^{5}$ Joo-Cheol Park ${ }^{6}$ Jan $\mathrm{Hu}^{7}$ James Simmer ${ }^{7}$ Jung-Wook Kim ${ }^{1,3}$ iD}

${ }^{1}$ Department of Pediatric Dentistry \& Dental Research Institute, School of Dentistry, Seoul National University, Seoul, Korea

${ }^{2}$ Department of Pedodontics, Faculty of Dentistry, Istanbul University, Istanbul, Turkey

${ }^{3}$ Department of Molecular Genetics \& Dental Research Institute, School of Dentistry, Seoul National University, Seoul, Korea

${ }^{4}$ Department of Pediatric Dentistry, Faculty of Dentistry, University of Eskisehir Osmangazi, Eskisehir, Turkey

${ }^{5}$ Seoul Chungdam Children's Dental Center, Seoul, Korea ${ }^{6}$ Department of Cell and Developmental Biology \& Dental Research Institute, School of Dentistry, Seoul National University, Seoul, Korea

${ }^{7}$ Department of Biologic and Materials Sciences, School of Dentistry, University of Michigan, Ann Arbor, MI, USA

\section{Correspondence}

Jung-Wook Kim, Department of Molecular Genetics, Department of Pediatric Dentistry \& Dental Research Institute, School of Dentistry, Seoul National University, 101 Daehak-ro, Jongno-gu, Seoul 03080,

Korea.

Email:pedoman@snu.ac.kr

\section{ORCID}

Jung-Wook Kim (iD https://orcid.org/0000-0002-9399-2197

\section{REFERENCES}

Beattie, M. L., Kim, J. W., Gong, S. G., Murdoch-Kinch, C. A., Simmer, J. P., \& Hu, J. C. (2006). Phenotypic variation in dentinogenesis imperfecta/dentin dysplasia linked to 4q21. Journal of Dental Research, 85, 329-333.

Kawasaki, K., \& Weiss, K. M. (2006). Evolutionary genetics of vertebrate tissue mineralization: The origin and evolution of the secretory calcium-binding phosphoprotein family. Journal of Experimental Zoology Part B: Molecular and Developmental Evolution, 306, 295-316.

Kim, J. W., \& Simmer, J. P. (2007). Hereditary dentin defects. Journal of Dental Research, 86, 392-399.

McKnight, D. A., Simmer, J. P., Hart, P. S., Hart, T. C., \& Fisher, L. W. (2008). Overlapping DSPP mutations cause dentin dysplasia and dentinogenesis imperfecta. Journal of Dental Research, 87, 1108-1111.

McKnight, D. A., Suzanne Hart, P., Hart, T. C., Hartsfield, J. K., Wilson, A., Wright, J. T., \& Fisher, L. W. (2008). A comprehensive analysis of normal variation and disease-causing mutations in the human DSPP gene. Human Mutation, 29, 1392-1404.

Shields, E. D., Bixler, D., \& El-Kafrawy, A. M. (1973). A proposed classification for heritable human dentine defects with a description of a new entity. Archives of Oral Biology, 18, 543-553.

von Marschall, Z., Mok, S., Phillips, M. D., McKnight, D. A., \& Fisher, L. W. (2012). Rough endoplasmic reticulum trafficking errors by different classes of mutant dentin sialophosphoprotein (DSPP) cause dominant negative effects in both dentinogenesis imperfecta and dentin dysplasia by entrapping normal DSPP. Journal of Bone and Mineral Research, 27, 1309-1321.

Yamakoshi, Y., Nagano, T., Hu, J. C., Yamakoshi, F., \& Simmer, J. P. (2011). Porcine dentin sialoprotein glycosylation and glycosaminoglycan attachments. BMC Biochemistry, 12, 6.

Yamakoshi, Y., \& Simmer, J. P. (2018). Structural features, processing mechanism and gene splice variants of dentin sialophosphoprotein. The Japanese Dental Science Review, 54, 183-196.

Yang, J., Kawasaki, K., Lee, M., Reid, B. M., Nunez, S. M., Choi, M., ... Hu, J. C. (2016). The dentin phosphoprotein repeat region and inherited defects of dentin. Molecular Genetics \& Genomic Medicine, 4, 28-38. 